\begin{tabular}{ccc}
\hline International Journal of Engineering \& Technology, $7(3.8)(2018) 92-96$ \\
SPC & Website: $w$ ww. sciencepubco.com/index.php/IJET \\
Research paper & Technology \\
\hline
\end{tabular}

\title{
Detection of Optical Characters Using Text Structure Modeling
}

\author{
Miss Velantina Kapale ${ }^{I}$, Prof. Mangesh Ghonge ${ }^{2}$ \\ ${ }^{1}$ Student, M.E.(Computer), SITRC, Nashik \\ ${ }^{2}$ Assistant Professor, Computer Department, SITRC, Nashik \\ lvelantinak@gmail.com \\ ${ }^{2}$ mangesh.ghonge@sitrc.org
}

\begin{abstract}
The basic use of back propagation(bp) neural network for textual content categorization and category in addition to type and could gather high everyday giant overall performance and accuracy. But, the high-quality drawback of this network is its prolonged training time. The genetic algorithm is regularly used to generate weights on network layer and beneficial for optimization. In proposed system we employ the mixture of each genetic set of rules and the returned propagation neural community for text categorization additionally the usage of the textual content structure we will understand the unique enter object through using characteristic extraction input item. We're able to use the genetic algorithm to reduce weights of edges within the but returned propagation neural community in used to backpropagating techniques. On the equal body, we are able to used to enhance the genetic algorithm to increase its performance and accuracy of neural network.
\end{abstract}

Keywords: Text categorization, Back propagation, BPNN, Genetic algorithm, Neural network.

\section{Introduction}

Optical character recognition (OCR) programs are the substantially performed in numerous domain names to transform hardcopy documents into digital documents for higher garage, vital tenancy, are seeking out capability, better get proper of entry to moreover understand of digital characters[ 7].

Utilization of the neural networks for photo processing advanced specially because of improvement in technology that allowed improvement of algorithms for schooling neural networks and additionally creates the complex featured neural community architectures and blueprint of the community. Artificial neural network is can be utilized in the OCR packages as smooth classifiers or as combination of feature extractor and classifiers and art work in every the strategies which is probably defined[8].

The important thing disadvantage of the lower back- propagation again is that it does now not guarantee that the neural community will converge to a worldwide minimal. several strategies are furnished inside the literature that may be used over the as soon as returned-propagation to in show the convergence of the neural network, like gradient-based studying getting to know and splendid reading techniques, utilizing distortions to the pattern from running out set, determine even as the learning price must without a doubt be modified[11][16].

The text categorization hassle is probably defied as following: several documents and classes are made and we should classify those files to a number of the ones education in step with their contents. This situation has been appreciably researched these days. Many of the of techniques that have already been proposed for textual content categorization. For the cause that writing categorization hassle is a high measurement and non-linear problem, it's appropriate for a right away decrease returned propagation neural network (BPNN) to treatment the trouble. Irrespective of the reality that this version isn't interpretable, it may advantage excessive performance and excessive dimensionality.

But, the top notch disadvantage of a BPNN is the extended schooling time. In a BPNN, it calls for sort of years to back- propagate and regulate weights of connections or can edges of links. And this approach may additionally want to frequently repeat numerous instances. The overall performance training time imposed guidelines on packages and capabilities of the BPNN.

We use the genetic set of rules to optimize the BPNN and use the optimized BPNN for text categorization reason in addition that processing and get efficient very last effects. On the same time frame, to decorate the effectiveness of the genetic set of rules, we advanced the identical general genetic algorithm. The consequences suggests that our method perform plenty better than the identical standard method it truly is frequently on the cornerstone of the BPNN.

Computational call for of genetic set of rules get maximize together with the size of the search area, it is the number one disadvantage of the genetic set of rules. Therefore, returned propagation based totally absolutely techniques receives the first threat to education dataset for the immoderate dimensional are looking for regions and it can art work in long schooling set additionally[19].

\section{Literature Survey}

Peter Bell, Member, IEEE, et al [1], Using hybrid DNN structures with clustered triphone dreams is now general in computerized speech recognition. however, The usage of a unmarried set of DNN objectives on this way might not be the most effective 
choice, for the motive that desires are the quit result of a especially arbitrary clustering method that most appropriate be ideal for discrimination.

Yonghao he, Shiming xiang, et al [2], generally, From many one of the maximum essential subjects considered thru the similarity characteristic is a way to make the model similarity computable. By reason of the robust development of deep studying in computer belief and prescient and herbal language processing, it's miles replicable of extract semantic representations from each photograph and text facts thru the usage of the use of deep neural networks.

Kun li, Xiaojun Qian, and Helen Meng [3], the ERNs leverages present day-day automatic speech recognition era through constraining the quest area via collectively with the probably phonetic errors sample of the aim terms further to the canonical transcriptions. Hints are professional independently and as a end result contextual records is out of place.

Hamid Palangi, et al [4], The automatic key-word detection and difficulty depend allocation competencies enabled via manner of manner of manner of the LSTM-RNN permit the network to perform record retrieval, a hard language processing challenge, wherein the similarity some of the query and documents can be measured via the distance among their corresponding sentence embedding vectors is computed and calculated with the beneficial aid of the LSTM-RNN.

Hao Wang, et al [5], For better-diploma, probabilistic graphical models using their Bayesian sequence nature are despite the fact that that more bendy. To gather covered intelligence which includes both perception and inference, it is quite a distance glaring ly relevant to tightly combine deep learning and Bayesian fashions within the principled probabilistic framework, which we can say in Bayesian deep community.

Xiaohang Ren, Yi Zhou, et al [7], the CNN version is pre-skilled through a convolutional sparse vehicle-encoder (case) in an unmonitored manner to assist extracting complex Chinese language text capabilities from herbal photos and enlarging the training set. The text shape issue detector (TSCD) layer, which includes several text shape detail detectors, is mainly designed for extracting Chinese language text structure features.

Each of the textual content form trouble detectors is designed to extract the excellent skills of remarkable forms of Chinese language language person shape additives and S. M. Lucas, et al [8], explains to benefit a smooth image of the nation of work of analyzing text in scenes furthermore of the consequences using favored techniques of vicinity precision/recall into account in thoughts and edit distance given with the aid of a. Shahab, F. Shafait, and a. Dengel [9].

Lingxi Xie, Alan Yuille [11], MDD are done with the beneficial useful resource of comparing the diagnosed transcriptions with the canonical ones. Regardless of the reality that this technique performs reason-ably nicely, it's the following problems: (1) studying the error patterns of the target phrases to generate the ERNs stays a hard work.

Telephones or smart phone mistakes omitting from the ERNs cannot be identified irrespective of the truth we have been received well-professional acoustic models; (2) using the properties of acoustic models and phonological modeling approach the deep convolutional neural network (CNN) version is big-scale visual recognition. comply within essential thoughts which includes growing the depth and constructing dual carriageway connections, re- searchers have manually de-signed an extension of constant net- paintings structures and verified their effectiveness, furthermore an encoding technique used to symbolize each community shape with a hard and fast-duration binary string, the famous genetic operations which incorporates mutation or crossover to find out the search place correctly.

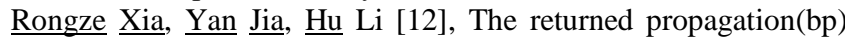
neural community broadly hired for textual content categorization will also advantage excessive famous overall performance. And extra over the usage of the genetic sequence of is frequently generate answers for optimization. Multilayered feed ahead neural networks personal a number of homes which cause them to in particular appropriate to complicated sample classification troubles.

David J. Montana and Lawrence Davis [13], But, their software to actual-global problems has been hampered thru the shortage of an exercising algorithm which reliably unearths a completely almost globally maximum fulfilling requirement. Genetic algorithms are a form of optimization techniques which are appropriate at exploring a large and complicated location in a smart manner to get values close to the worldwide maximum suitable values of the node. Lucian-Ovidiu Fedorovici, Raduemil Precup, et el.[15], The gravitational are looking for for algorithms (GSAS) are finished in collaborate with lower back propagation (BP) algorithm as optimization algorithms in working out way of a specification CNN form for OCR programs.

Mengjie Zhang and Victor Ciesielski[16], Pixel based totally definitely neural networks educated with the beneficial useful resource of the over again propagation and genetic algorithms at the hassle of locating small key points in massive images.

In level one, the networks are professional by manner of once more- ward mistakes propagation or the first genetic algorithm with the health of regular sum squared errors on lessen-outs of the objects of hobby.

Dangle Zhuang, Changlong Li, Qingfeng Wang, et el. [17], system indicates the leverage stroke for analyzing the non-save you instance of Chinese language Language characters. Primarily based at the techniques of convolution neural form a natural language processing company for Chinese language textual content classification.

Rui Liu, Minghu Jiang [19], A gravitational seek algorithm is implemented in function preference to over come a crucial bias of the non balanced function set also we are able to accumulate immoderate terrific classifications and an extended manner loads much less time ingesting than one in each of a type neural networks, which includes as quickly as once more propagation (bp) community and radial foundation feature (RBF) community, and so forth.

Lichao Mou, et al [20], a unique RNN version that can correctly study high spectral pixels as sequential records after which determine records training via community reasoning. RNN frame-work has been proposed for the excessive spectral picture classification model.

\section{System Architecture/ System Overview}

Problem Statement: To come across optical characters from images the usage of the convolutional neural community primarily based totally absolutely category method the usage of the techniques of lower again propagation and genetic algorithm to restrict the computation furthermore to optimization of community. Deep mastering based totally honestly strategies educate a deep community to extract talents in update of the guideline designed function extractors, which may be difficult to optimize for textual content detection. Convolutional neural community ( $\mathrm{CNN})$ is the maximum famous deep learning models for textual content detection.

The art work in trains a five-layer CNN version to discover text regions in herbal images thru the use of a supervised grasping technique. Moreover teach a $\mathrm{CNN}$ version with convolutional layers to encounter text regions in herbal photographs. The use of the first convolutional layer is pre-trained and categorized with an SVM classifier.

Maximally stable outside area (MSER) is used as a candidate textual content location extractor to lessen the wide shape of information regions in advance than the CNN model.

\section{System Analysis}

\subsection{Proposed System}


We describe a completely specific genetic algorithmic set of for schooling feed forward networks. It not most effective succeeds in its challenge however it outperforms back propagation, the same old training set of rules. On example.

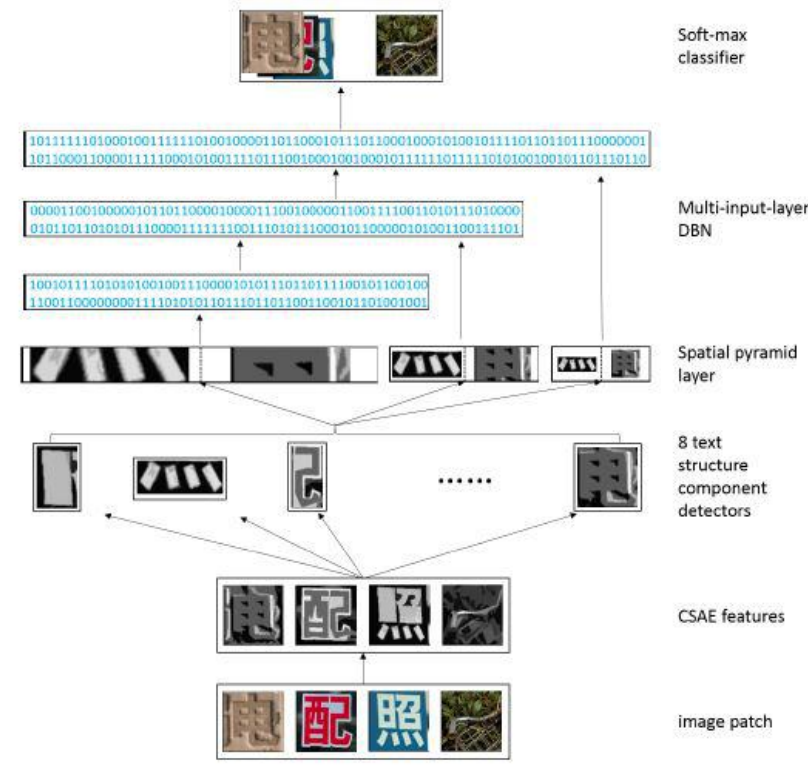

This fulfillment comes from tailoring the genetic algorithm to the area of schooling neural networks. Shown in figure 2, for each textual content shape thing group, a selected TSCD is designed to extract its function, which has its specific characteristic map form. The multi-form function maps within the TSCD layer moreover re-strict the similarity of capabilities whilst the feature map size expands for this reason the requirement of training set is reduced. In existing device, texture-based totally approaches come across texts by using their special texture systems and normally use gadget mastering strategies to distinguish texts from history by means of extracting certain functions. as a standard instance, Chen et al. layout the several susceptible classifiers that we get via using joint possibilities for characteristic responses and use Adaboost system learning set of rules to construct a robust classifier for detecting texts from furnished. Ye et al. use multi-scale wavelet remodel to extract features and an SVM classifier is applied to pick out the textual content traces from scene photos.

\section{Algorithms used in the proposed System:}

1) Neural Networks: Neural networks are algorithms for optimization and studying primarily based loosely on requirements stimulated through research to the characters or item of the brain. They normally include the 5 components of the community:

1. A directed graph referred to as town topology whose arcs we searching for the advice from as hyperlinks.

2. A kingdom of variables is related to every node.

3. A real-valued weight written by genetic algorithm is related to each link.

4. A real-valued is the unfairness linked to each node of community.

5. A transfer the function for every point determines state of a node as a feature of a) its bias b, b) the weights, wt of its incoming links, and c) the states, $\mathrm{x}$, of the nodes related to it via the ones hyperlinks. This switch feature commonly lakes the proper execution wherein / is whether sigmoid or a step characteristic. A feed forward community is one whoso topology does not have the closed paths.

A feed forward network is one whoso topology does no longer have the closed paths. Its input nodes are those without a arcs for them, and its output nodes don't have any arcs a protracted way from them. All of the nodes are hidden nodes. At the equal time since the states of all input nodes are set, all of other nodes in the community may also set their states as values propagate through the community. The operation of a feed forward network incorporates calculating the outputs given a difficult and rapid processing of inputs on this manner. This kind of form of neural network is connected with the node via node, if every node in layer $i$ is hooked up to any or all different nodes in layer i+l 1 or all $\mathrm{i}$.

2) Genetic Algorithms: Genetic algorithms are algorithms for optimization and mastering based on the whole loosely on numerous competencies of natural evolution. They require the five additives:

1. A forms of encoding solutions of given problem of network to the problem on chromosomes.

2. A exam feature that returns a score each chromosome directed at it.

3. A manner of initializing the population of chromosomes

4. Operators which can be placed on if they re-produce to adjust their genetic composition. Blanketed is probably mutation, crossover (that is recombination of genetic values), and do-principal-specific operators.

5. Parameters settings for algorithm, operators, and so on for in addition class guidelines.

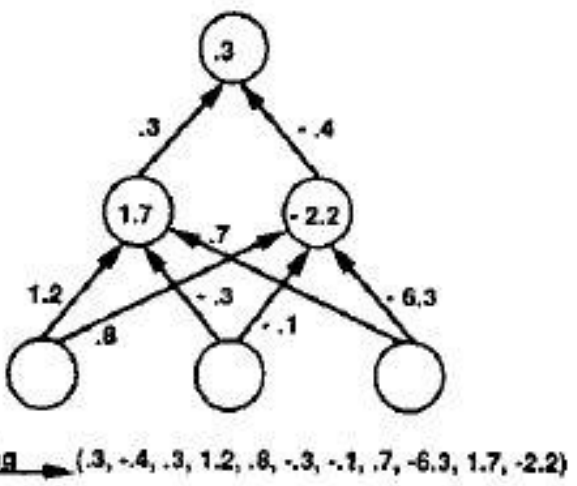

Fig. 3. Encoding Network on a Chromosome

$$
(3,-.4,1.2,8,-3, \cdot, 1,7,-6.3)
$$

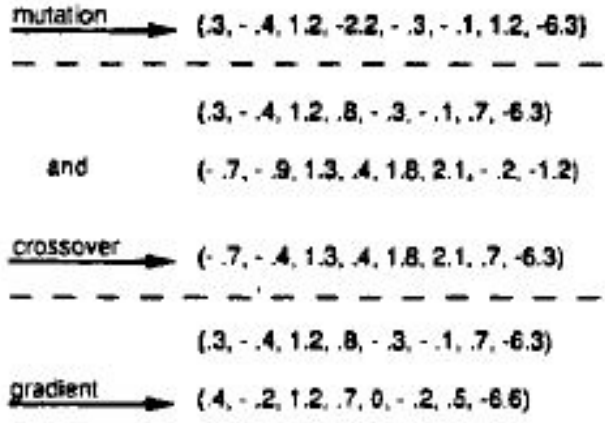

Fig. 4. Operation of the Operators

Given those five components, a genetic asset of regulations operates according to next steps are as follows:

1. Population is initialized, making use of the device in c3. The out-come of initialization is a few chromosomes values as determined in $\mathrm{c} 2$.

2. Number of the population are valued of genetic set of rules is evaluated, utilizing the function in $\mathrm{c} i$. opinions might be normalized; the essential factor is to steadfastly hold up relative rating of opinions.

3. The populace undergoes duplicate until a stopping criterion is met. duplicate consists of numerous iterations of the following 3 steps: 
- Greater mother and father are selected to reproduce. Preference is stochastic, however the dad and mom with the high-quality evaluations are preferred within the decision the parameters of 55 can impact the decision giver.

- The operators of c4 are applied primarily based onto the figure's operator to supply kid's operator. The parameters of c5 assist decide which operators to apply.

- $\quad$ The kid's operator are evaluated and inserted to the populace or most important price operator. In some variations of the genetic set of guidelines, the complete populace is resituated in each cycle of reproduction of the schooling dataset network layer. In others, maximum reliable subsets of the population are changed by means of new fee characteristic.

Right away as a genetic set of regulations is administered the usage of a illustration that use-absolutely encodes answers to an problem and operators which 4 can also generate better people from unique parents, algorithm can seasoned-duce populations of better and better feature of given form of the network, converging the eventually on effects near to a global maximum excellent. In lots of instances (together with the instance referred to on this paper), the same kind of operators, mutation and crossover, are enough for performing the optimization. In such instances, genetic algorithms can characteristic a black-field feature optimizer now not requiring their writer to enter any understanding about the area. However, as illustrated on this paper, know-how of the vicinity can regularly be exploited to beautify the genetic algorithms considerable overall performance via the incorporation of latest operators.

\subsection{Mathematical Model}

Neural Network algorithms - Mathematical representation: System Description:

- Input: we take three inputs, $\mathrm{x} 1, \mathrm{x} 2, \mathrm{x} 3$

- Output: w1,w2,..w1,w2,..., real numbers expressing the valuable of respective inputs to output.

- Through education and testing dataset we find the accuracy of dataset and calculate the succession.

\section{Mathematical formulation:}

$\mathrm{S}=\{\mathrm{X}, \mathrm{W}, \mathrm{O}\}$

Where,

$\mathrm{X}=$ Perceptron with input set

$=\sum_{n=1}^{n} \mathrm{Xi}=\{\mathrm{x} 1, \mathrm{x} 2, \mathrm{x} 3, \ldots \ldots \ldots, \mathrm{xn}\}$

Where $\mathrm{n}>0$

$=$ ex. Binary Input set

$\mathrm{W}=$ Weights

$=\sum_{n=1}^{n} w i=\{w 1, w 2, w 3$

Where $\mathrm{n}>0$

$=$ ex. $\mathrm{w} 1, \mathrm{w} 2, . . \mathrm{w} 1, \mathrm{w} 2, \ldots$, real numbers expressing significance of respective inputs to output.

$\mathrm{O}=$ neuron's output, 0 or 1

$=\sum_{j}^{0} w j x j$

Where $\mathrm{j}>0$
$=$ function value is less than or more than some threshold value

\subsection{System Design}

We hire the back mistakes propagation set of rules and a genetic set of rules with help from health of favored summation squared mistakes to train pixel primarily based honestly neural networks technique and strategies on the sub pictures (cutouts) within the first degree, and art work with a second genetic algorithm with help of the fitness function and heuristic feature focused on precision and recall at the entire schooling images dataset for the refinement of every of the professional networks above within the $2^{\text {nd }}$ stage.

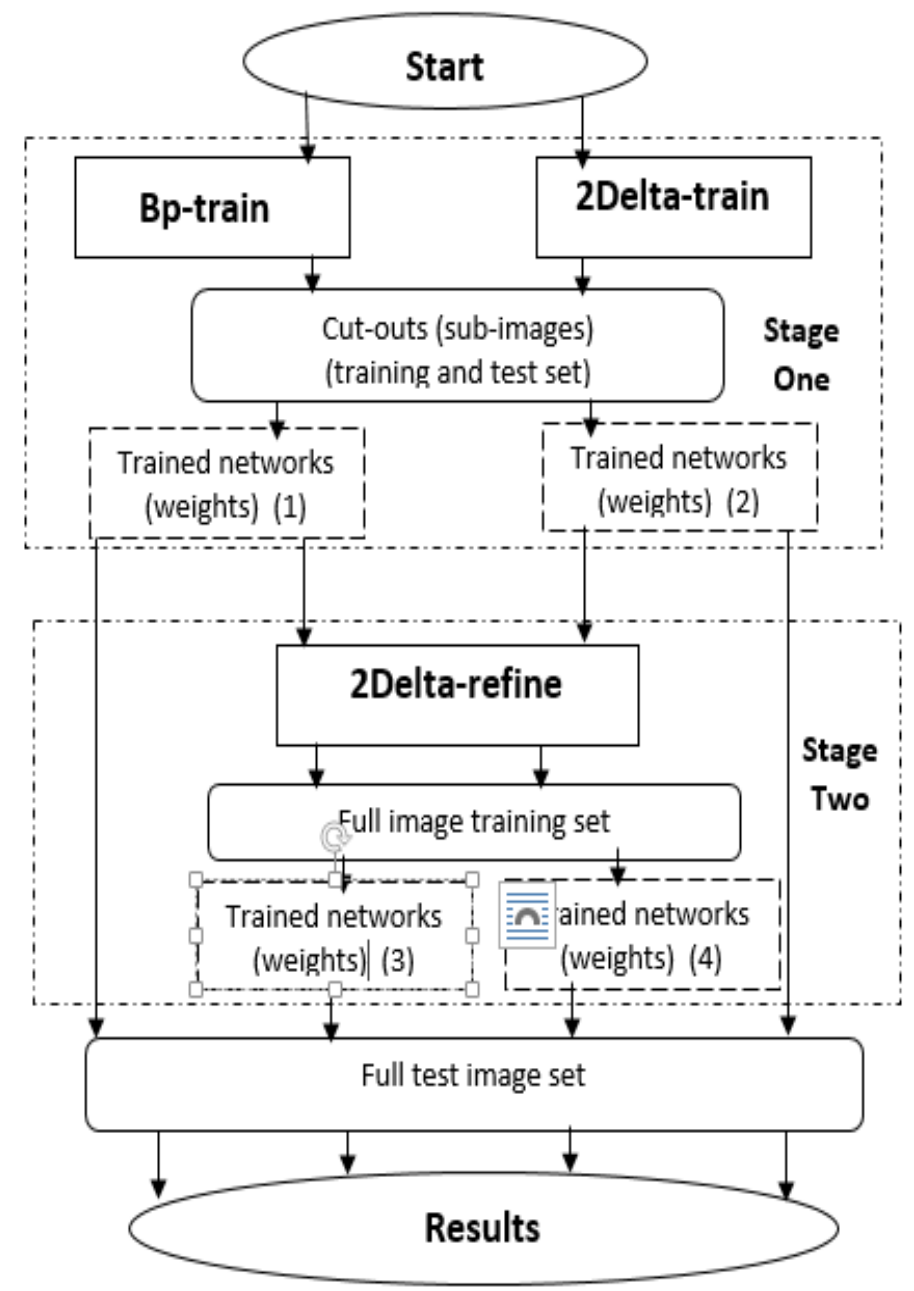

The 2nd techniques within the first stage can be used to gather the final outcomes for object detection primarily based mostly on the essential item detection device the usage of various strategies of it. Readily, we call the two strategies BP train and 2delta- train respectively. For the second one genetic algorithm in the 2nd stage, we name it 2delta-refine to inform aside the primary one within the first degree. Because of this, distinct aggregate of the two tiers will result in new methods for object detection: BP-teach + 2deltarefine and 2delta-train +2 delta-refine[16].

\section{Performance Analysis}

\section{A. Dataset}

For checking out cause static picture dataset jointly to be had online. At this present day position many dataset available for plenty languages. We have the now to start with we've taken one particular language dataset to test our module that is available online. 


\section{B. Implementation}

Feature extraction: first stage is mastering the tokens, after carry outing the gaining knowledge of the tokens characteristic extraction is completed. Taking the photograph as a enter it's far first step of detecting the OCR of particular language in function extraction. After giving the enter to device with the assist of imply as well as preferred deviation capabilities are extracted from the given word. It indicates studying and detection via scanned individual in the end result.

\section{Result}

The end result indicates that the OCR from the given enter pictures i.e. dataset of pix, it could be massive in amount and algorithmic seasoned apply at the dataset and device will supply the correct result. Algorithm used in this machine generate correct and powerful bring about minimal quantity of time.
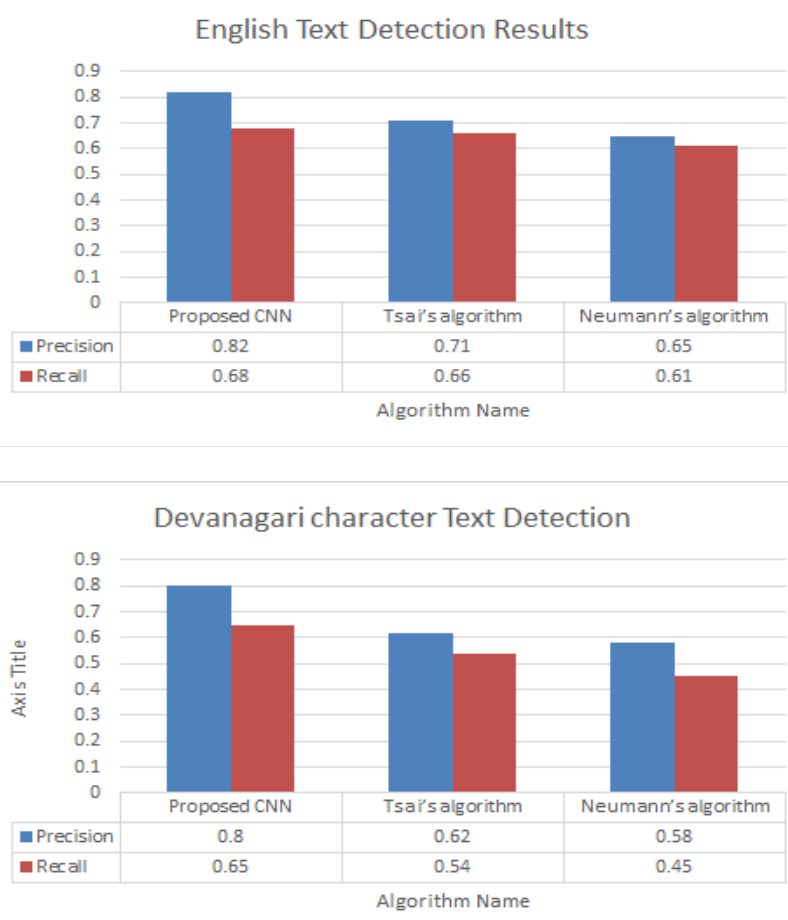

\section{Conclusion}

Back propagation (BP) neural network is substantially used for textual content categorization and will gather immoderate overall performance. But, the finest downside of this network is its lengthy schooling time. The genetic set of policies is often used to generate useful solutions for optimization. In proposed gadget we are going to com-bine the genetic set of regulations and the decrease returned propagation neural network for textual content categorization. We are able to use the genetic set of regulations to optimize weights of connections within the returned propagation neural network in preference to conventional once more propagating. And, we're going to beautify the genetic set of rules to increase its performance. Also, we are going to conquer the traditional downside of the BP neural community. In proposed gadget we're in a position to reveal that our approach outperforms better than the conventional method for text categorization in order that processing pace of network is expanded.

\section{Acknowledgement}

I would sincerely like to thank our Professor Mangesh Ghonge, Department of Computer Engineering, SITRC, Nashik for his guidance, encouragement and the interest shown in this project by timely suggestions in this work. His expert guidance as well as recommendation feedback had greatly enhanced effectiveness of this work.

\section{References}

[1] Peter Bell, Member, IEEE, Pawel Swietojanski, Member, Ieee, And Steve Renals, Fellow, Ieee,"Multitask Learning Of ContextDependent Targets In Deep Neural Network Acoustic Modls",Ieee/Acm Transactions On Audio, Speech, And Language Processing,2016 Ieee,Pp 1-10.

[2] Yonghao He, Shiming Xiang, Cuicui Kang, Jian Wang, And Chunhong Pan, Member, Ieee,’Cross-Modal Retrieval Via Deep And Bidirectional Representation Learning",Ieee Transactions On Multimedia, 2016 Ieee,Pp 1-15.

[3] Kun Li, Xiaojun Qian, And Helen Meng,"Mispronunciation Detection And Diagnosis In L2 English Speech Using Multi-Distribution Deep Neural Networks", Ieee/Acm Transactions On Audio, Speech, And Language Processing, 2016 Ieee,Pp 1-15.

[4] Hamid Palangi, Li Deng, Yelong Shen, Jianfeng Gao, Xiaodong He, Jianshu Chen, Xinying Song, Rabab Ward,'Deep Sentence Embedding Using Long Short-Term Memory Networks: Analysis And Application To Information Retrieval", Ieee/Acm Transactions On Audio, Speech, And Language Processing, 2015 Ieee,Pp 1-16.

[5] Hao Wang, Dit-Yan Yeung Senior Member, Ieee,'Towards Bayesian Deep Learning: A Framework And Some Existing Methods",Ieee Transactions On Knowledge And Data Engineering, 2016 Ieee,Pp $1-14$.

[6] Rui Zhao And Kezhi Mao,"Topic-Aware Deep Compositional Models For Sentence Classification,Ieee/Acm Transactions On Audio, Speech, And Language Processing",Journal Of Latex Class Files, Vol 13, No. 9, September 2014,Pp 1-12.

[7] Xiaohang Ren, Yi Zhou, Jianhua He, Senior Member, Ieee, Kai Chen Member, Ieee, Xiaokang Yang, Senior Member, Ieee, And Jun Sun Member, Ieee,A "Convolutional Neural Network Based Chinese Text Detection Algorithm Via Text Structure Modeling",Ieee Transactions On Multimedia,2016 Ieee, Pp 1- 14

[8] S. M. Lucas, A. Panaretos, L. Sosa, A. Tang, S. Wong, And R. Young, "Icdar 2003 Robust Reading Competitions", In Document Analysis And Recognition, 2003. Proceedings. Seventh International Conference On. Ieee, 2003, P. 682.

[9] A. Shahab, F. Shafait, And A. Dengel, Icdar 2011 Robust Reading Competition Challenge 2: Reading Text In Scene Images", In Document Analysis And Recognition (Icdar), 2011 International Conference On. Ieee, 2011, Pp. 14911496.

[10] Dimosthenis Karatzas, Sergi Robles, Lluis Gomez, "An On-Line Platform For Ground Truthing And Performance Evaluation Of Text Extraction Systems", Pp 1-5.

[11] Lingxi Xie, Alan Yuille, Genetic Cnn,4 Mar 2017,1-10.

[12] Rongze Xia, Yan Jia, Hu Li, 'Icdar 2003 Robust Reading Competitionsa Bp Neural Network Text Categorization Method Optimized By An Improved Genetic Algorithm", 2013 Ninth International Conference On Natural Computation (Icnc), 2013 Ieee, Pp 257-261.

[13] David J. Montana And Lawrence Davis, "Training Feedforward Neural Networks Using Genetic Algorithms", Pp 762-767.

[14] Majid Mohammadi And Mohammad Ali Mehrolhassani, "New Learning Approach By Genetic Algorithm In A Convolutional Neural Network For Pattern Recognition", Pp 1-13.

[15] Lucian-Ovidiu Fedorovici, Radu-Emil Precup, Et El., Embedding Gravitationa Search Algorithms In Convolutional Neural Networks For Ocr Applications, 7th Ieee International Symposium On Applied (Omputational Lntelligence And Informatics,May 24-26, 2012,Pp 125- 131.

[16] Mengjie Zhang And Victor Ciesielski, Using Back Propagation Algorithm And Genetic Algorithms To Train And Refine Neural Networks For Object Detection, December 8, 1998, Pp 1-12.

[17] Hang Zhuang, Changlong Li, Qingfeng Wang, Et El., "Natural Language Processing Service Based On Stroke-Level Convolutional Networks For Chinese Text Classification", 2017 Ieee 24th

International Conference On Web Services, 2017 Ieee, Pp 404-411.

[18] D. Karatzas, F. Shafait, S. Uchida, M. Iwamura, S. R. Mestre, J. Mas, D. F. Mota, J. A. Almazan, L. P. De Las Heras Et El., Icdar 2013 Robust Reading Competition, In Document Analysis And Recognition (Icdar),2013 12th International Conference On. Ieee, 2013, Pp. 1484-1493.

[19] Rui Liu, Minghu Jiang, "Chinese Text Classification Based On The Bvb Model", Fourth International Conference On Semantics, Knowledge And Grid, 2008 Ieee, Pp 376-379.

[20] Lichao Mou, Student Member, Ieee, Pedram Ghamisi, Member, Ieee,Et El, "Deep Recurrent Neural Networks For Hyperspectral Image Classification", Ieee Transactions On Geoscience And Remote Sensing, 2017 Ieee, Pp 1-17. 